\title{
Avaliação da Performabilidade do Sistema Ferroviário da Região do Recife
}

\author{
Performability Evaluation of the Rail System of Recife Region \\ Rodemarck Melo dos Santos Júnior, Gustavo Rau de Almeida Callou, \\ Osvaldo Marinho dos Santos Neto
}

Universidade Federal Rural de Pernambuco, Pernambuco, Brasil.

\begin{tabular}{l} 
I N F O A R T I GO \\
\hline Palavras-chave: \\
Sistema Ferroviário, \\
Avaliação de desempenho \\
disponibilidade, \\
Redes de Petri \\
Estocásticas, \\
Diagramas de Bloco de \\
Confiabilidade.
\end{tabular}

A R T ICLE INFO

\section{Keywords:}

Rail System,

Performance Evaluation

Availability,

Stochastic Petri Net,

Reliability Block

Diagrams.

\begin{abstract}
RESUMO
O sistema ferroviário da região do Recife possui capacidade para transportar aproximadamente 400 mil passageiros diariamente. Falhas diminuem drasticamente sua capacidade de transporte. Um exemplo de falha que ocorre frequentemente é a denominada falha de falsa ocupação. Falhas de falsa ocupação ocorrem quando o mecanismo de detecção de trens de parte do percurso apresenta defeito, obrigando o maquinista a assumir o controle manual, aumentando os riscos de acidentes. Uma das soluções é aumentar a capilaridade do sistema ferroviário a partir da adição de rotas alternativas. Nesse contexto, este trabalho propõe um conjunto de modelos para avaliação de desempenho e disponibilidade de sistemas ferroviários. Um estudo de caso utilizando o sistema ferroviário de Recife foi utilizado para mostrar a aplicabilidade dos modelos propostos, onde foi possível aumentar a disponibilidade do sistema em praticamente $50 \%$ com a adição de rotas alternativas, melhorando também as métricas de desempenho.

\section{A B S T R A C T}

The rail system in the Recife region can transport approximately 400,000 passengers daily. Failure drastically decreases its carrying capacity. An example of a failure that occurs frequently is the so-called false occupancy failure. False occupancy failures occur when the train detection mechanism for part of the route is defective, forcing the driver to take manual control, increasing the risk of accidents. One of the solutions is to increase the capillarity of the railway system by adding alternative routes. In this context, this work proposes a set of models for evaluating the performability of railway systems. A case study using the Recife railway system was used to show the applicability of the proposed models, where it was possible to increase the system availability by practically $50 \%$ with the addition of alternative routes, also improving the performance metrics.
\end{abstract}




\author{
Correspondência para autores: \\ rodemarck.meloj@ufrpe.br (Santos JR, R.M.S.) (ORCID: 0000-0002-6050-9074), \\ gustavo.callou@ufrpe.br (Callou, G.R.A.) (ORCID: 0000-0002-7997-374X), \\ osvaldo.santosnt@ufrpe.br (Santos Neto, O.M.) (ORCID: 0000-0001-9373-8589)
}

\title{
1. Introdução
}

Com aproximadamente $71 \mathrm{~km}$ de extensão e transportando uma média de 400 mil passageiros diariamente, o sistema ferroviário da região metropolitana do Recife (RMR) é administrado pela Companhia Brasileira de Trens Urbanos (CBTU) através da Superintendência de Trens Urbanos do Recife (METROREC) (CBTU, 2021). Devido a baixa capilaridade ferroviária um único defeito pode parar toda a frota de trens. Então, é crucial que aconteça o mínimo de falhas possível de qualquer natureza. Falhas causadas por falsas ocupações (FOs) são geralmente ocasionadas por um defeito nos equipamentos que fazem a detecção da presença dos trens na ferrovia. Esse tipo de falha é crítico, uma vez que a detecção errada de um trem em um circuito de via (CDV) impede que os demais trens possam seguir seu trajeto. Quando a falha causada por uma FO é identificada, o trem pára de contar com o controle automatizado e, assim, o maquinista assume o controle manual do trem. Esse trem passa a circular em baixa velocidade.

A fim de evitar ou reduzir esse tipo de problema das FOs, este artigo propõe uma estratégia baseada em modelos para maximizar a disponibilidade dos equipamentos que compõem a malha ferroviária. Por exemplo, a estratégia adotada é capaz de identificar os CDVs que mais têm gerado falhas. A modelagem em diagrama de blocos de confiabilidade (RBD) é utilizada para detectar tais CDVs e, assim, é possível colocar redundância para maximizar a disponibilidade do sistema. Esse trabalho também tem como objetivo o de representar o funcionamento da malha ferroviária através das redes de Petri estocástica (Stochastic Petri Net (SPN)) para que se possa quantificar métricas de desempenho. Além disso, também é possível quantificar o impacto da melhora da disponibilidade no desempenho do sistema analisado.

O restante deste trabalho está organizado como descrito a seguir. A Seção 2 apresenta a fundamentação teórica necessária para um maior entendimento do trabalho. A Seção 3 mostra trabalhos relacionados a essa pesquisa. A Seção 4 apresenta a metodologia proposta para avaliar os sistemas ferroviários. Em seguida, a Seção 5 apresenta os modelos propostos para representar os sistemas ferroviários e computar as métricas de interesse como, por exemplo, disponibilidade e tempo de viagem. A Seção 6 ilustra a aplicabilidade dos modelos propostos através da análise do sistema ferroviário da cidade de Recife. Por fim, a Seção 7 apresenta as conclusões e os futuros direcionamentos desta pesquisa.

\section{Fundamentação Teórica}

\subsection{Redes de Petri Estocástica}

As redes de Petri estocásticas, stochastic Petri nets (SPN), são uma extensão das redes de Petri (MACIEL et al., 1996) e adicionam um tempo médio para disparo de uma determinada transição. São gerados vários valores de tempo diferentes e aleatórios que giram em torno do tempo médio para disparo, esses números seguem a distribuição exponencial. Desta forma, são adicionados dois novos tipos de transições que são as transições temporizadas, que seguem uma distribuição exponencial, e as transições imediatas, que têm 
o tempo zero para disparo. A Figura 1 mostra os componentes básicos das SPNs.

Figura 1: Componentes adicionados a rede de Petri.
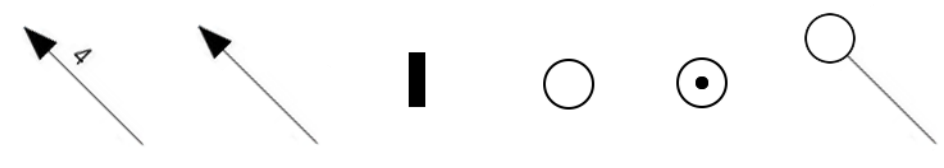

Arco ponderado

Arco direcionado Transição imediata

Lugar

Lugar com token

Arco Inibidor Transição exponencial

Como exemplo de utilização de modelagem utilizando SPN, considere um ciclo simplificado para se tomar café. O estado inicial corresponde ao copo vazio, onde pode-se encher o copo com café passando para o estado de copo com café sem açúcar. Posteriormente, pode-se colocar açúcar e, assim, ir para o estado de copo com café com açúcar. Nesse estado, a ação de beber café faz com que se volte ao estado original do copo vazio.

A Figura 2 mostra o modelo SPN proposto para representar o sistema acima descrito. Esse modelo foi criado utilizando a ferramenta Mercury Tool (SILVA et al., 2015). No modelo, os lugares COPO_VAZIO, CAFE_SEM_ACUCAR E CAFE_COM_ACUCAR representam os estados que o sistema pode ter. Já as transições ENCHER_COPO, COLOCAR_ACUCAR e BEBER_CAFE representam as ações que fazem com que o sistema mude de estado. A marcação inicial foi representada com uma marca (token) no lugar COPO_VAZIO.

Figura 2: Modelagem em SPN usando Mercury tool.

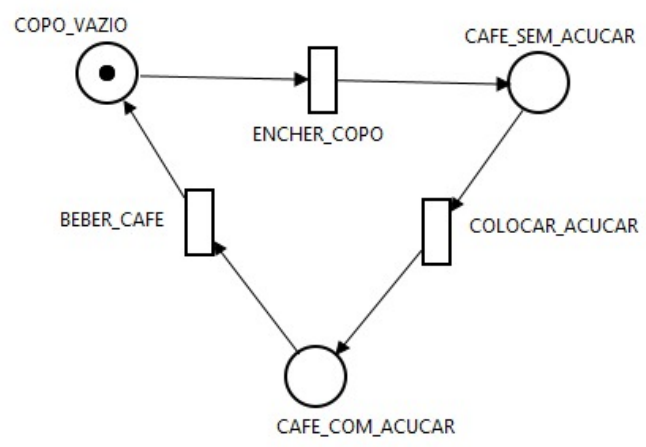

No modelo ilustrado na figura 2, partindo da marcação inicial, pode-se disparar a transição ENCHER_COPO. O disparo desta transição vai consumir um token do lugar COPO_VAZIO e gerar um token no lugar CAFE_SEM_ACUCAR. Após isso, a transição habilitada para ser disparada é a transição COLOCAR_ACUCAR. Ao disparar a transição COLOCAR_ACUCAR, o token de CAFE_SEM_ACUCAR é consumido, e um token é gerado em CAFE_COM_ACUCAR. A transição BEBER_CAFE pode então ser disparada. O disparo desta transição consome um token de CAFE_COM_ACUCAR e gera um token em COPO_VAZIO, voltando ao estado inicial.

É importante destacar que é possível adicionar um tempo médio para o disparo de cada transição. Sendo assim, pode-se, por exemplo, calcular o tempo que o ciclo leva para ser concluído ou calcular a disponibilidade de sistemas e/ou equipamentos.

Assumindo um outro modelo SPN com dois lugares definindo os estados ON e OFF, além de duas transições definidas como FAIL, que consome o token em ON e gera em OFF, e REPAIR, que consome o 
token de OFF para gerar em ON. A Equação 1 mostra como computar a disponibilidade desse sistema (MACIEL et al., 2012). Nessa equação, "E" representa a esperança que é a soma do produto de todas as possibilidades de marcações. Além disso, pode-se computar a disponibilidade sabendo a probabilidade de termos o sistema funcionando, ou seja, de termos um token no lugar ON. A Equação 2 mostra essa forma de se computar a disponibilidade.

$$
\begin{aligned}
& \frac{E\{\# O N\}}{E\{\# O N\}+E\{\# O F F\}} \\
& P\{\# O N=1\}
\end{aligned}
$$

Para se calcular o tempo necessário para ser completado um ciclo no modelo descrito na Figura 2, é necessário somar o tempo para cada ação. Esse tempo é representado pelos tempos associados a cada uma das transições do modelo. Esse resultado presume que nunca faltará café ou açúcar. Com a finalidade de representar a possibilidade de se ter a falta de um desses produtos, foi adicionado no modelo duas sub-redes para representar quando temos café disponível (token no lugar CAFETEIRA_ON) e açúcar disponível, token no lugar (ACUCAREIRO_ON), conforme pode ser visto na Figura 3. É importante destacar que também foram adicionadas as dependências de que a cafeteira não pode estar em OFF para disparar a transição ENCHER_COPO, e que o açucareiro não pode estar em OFF para disparar a transição COLOCAR_ACUCAR.

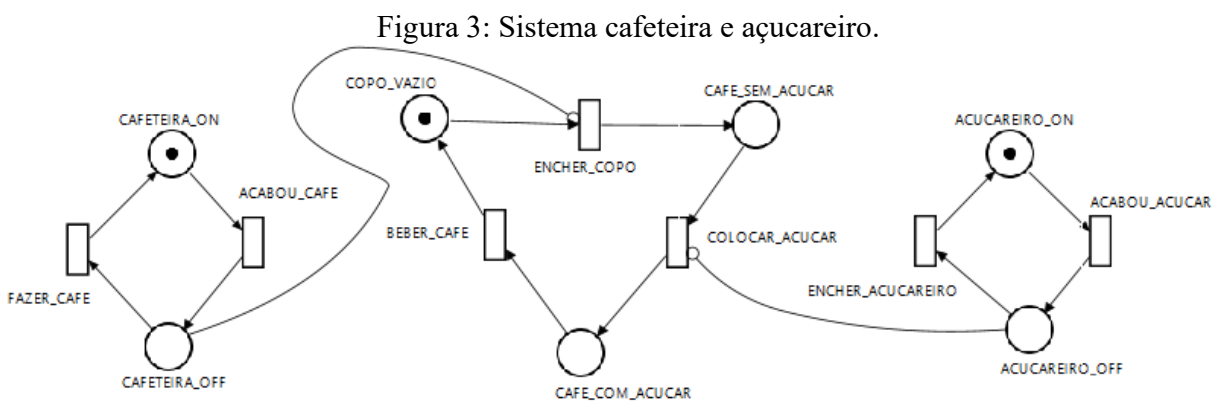

O modelo descrito na Figura 3 tornou mais complexo e difícil de se calcular o tempo necessário para completar o ciclo devido à adição de dependências. Todavia, como o modelo é um circuito fechado, com um número limitado de tokens, pode-se definir a velocidade média do circuito como sendo a soma da esperança de cada lugar pertencente ao circuito dividido pela divisão da esperança de um lugar P, pertencente ao circuito, pela vazão do lugar P. Desta forma, chega-se à Equação 3 que será utilizada para calcular o tempo demandado para se completar um ciclo completo. A Tabela 1 apresenta os parâmetros de entrada com os valores dos tempos associados a cada uma das transições do modelo. Após o cálculo pela utilização da Equação 1, podese obter a disponibilidade de $82 \%$ para a cafeteira e $94 \%$ para o açucareiro. A Equação 3 é utilizada para se obter o tempo médio para se completar um ciclo, no caso de 60 segundos.

$\frac{\left(\mathrm{E}\left\{\# \mathrm{COPO} \_ \text {VAZIO }\right\}\right)+\left(\mathrm{E}\left\{\# \mathrm{CAFE} \_\mathrm{SEM} \_ \text {ACUCAR }\right\}\right)+\left(\mathrm{E}\left\{\# \mathrm{CAFE} \_\mathrm{COM} \_ \text {ACUCAR }\right\}\right)}{\left(\frac{E\left\{\# C A F E \_C O M \_A C U C A R\right\}}{35}\right)}$ 
Tabela 1: Tempo médio de disparo em segundo.

\begin{tabular}{|l|c|}
\hline Transição & Tempo médio para disparo \\
\hline ENCHER_COPO & 10 \\
\hline COLOCAR_ACUCAR & 5 \\
\hline BEBER & 35 \\
\hline ACABOU_CAFE & 250 \\
\hline FAZER_CAFE & 50 \\
\hline ACABOU_ACUCAR & 400 \\
\hline ENCHER_ACUCAREIRO & 20 \\
\hline
\end{tabular}

\subsection{Diagramas de blocos de confiabilidade}

Os diagramas de bloco de confiabilidade, Reliability Block Diagrams (RBD), são redes de blocos conectados de forma a simular o impacto de falhas na disponibilidade. Em um diagrama RBD existe um vértice de entrada e um de saída, um conjunto de blocos que representam os componentes do sistema e arcos que ligam os blocos aos vértices (MACIEL et al., 2012). As principais informações que os blocos precisam para representar os componentes são, o tempo médio que o componente leva até apresentar um defeito e o tempo médio que o componente leva para ser recuperado, são respectivamente tempo médio de falha (MTTF) e o tempo médio de recuperação (MTTR). A Figura 4 apresenta uma modelagem em RBD de um sistema com 3 componentes ligados em série, representando que é necessário que os três componentes estejam disponíveis para que o sistema esteja disponível.

Figura 4: Modelagem em RBD com componentes em série.

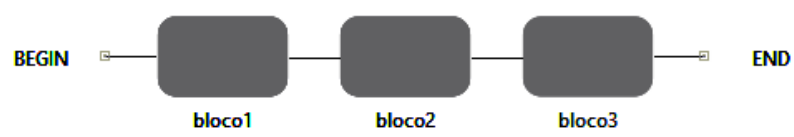

Em um diagrama RBD com $n$ blocos ligados em série, a probabilidade do sistema estar disponível pode ser definida como sendo o produto da probabilidade de cada bloco está disponível como mostrado na Equação 4, onde " $P$ " representa a probabilidade. A disponibilidade do sistema pode ser definida com o produto das disponibilidades de cada bloco como mostrado na Equação 5, onde " $D$ " representa a disponibilidade.

$$
\begin{gathered}
\prod_{i=1}^{n} P\left(b b_{0} c_{i}=1\right) \\
\prod_{n=1}^{n} D(b \text { bloco })
\end{gathered}
$$

A Figura 5 apresenta uma modelagem em RBD de um sistema com 3 componentes ligados em paralelo. Nessa representação é necessário que ao menos um dos componentes esteja disponível para que o sistema esteja disponível. Em um RBD com " $n$ " blocos em paralelo, a probabilidade do sistema estar disponível pode ser definido como o inverso do produto do inverso da probabilidade dos blocos estarem disponíveis como mostrado na Equação 6. Já a disponibilidade do sistema pode ser definida como o inverso do produto do inverso da disponibilidade dos blocos como mostrado na Equação 6.

$$
1-\left[\prod_{i=1}^{n} 1-P\left(b^{\prime} l o c 0_{i}=1\right)\right]
$$


Figura 5: Modelagem em RBD com componentes em paralelo.

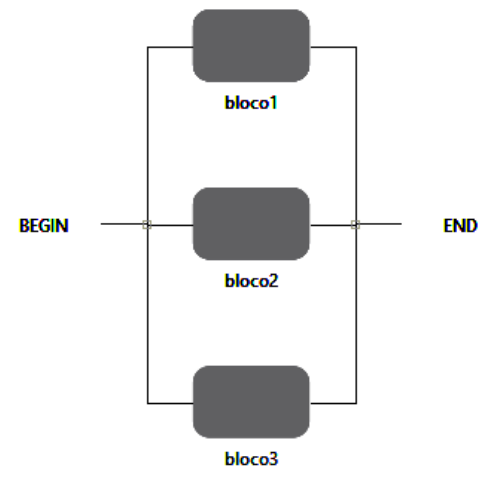

Para melhorar a disponibilidade do sistema representado na Figura 3, podemos adicionar uma segunda cafeteira. O modelo RBD correspondente é mostrado na Figura 6, onde o MTTR e MTTF de ambas as cafeteiras são respectivamente os valores "FAZER_CAFE" e "ACABOU_CAFE" e MTTR e MTTF do açucareira são respectivamente "ENCHER_ACUCAREIRO” e ACABOU_ACUCAR que estão na Tabela 1. A Equação 8 pode ser utilizada para calcular a disponibilidade do sistema que é de $\approx 92 \%$.

Figura 6: Sistema cafeteira dupla.

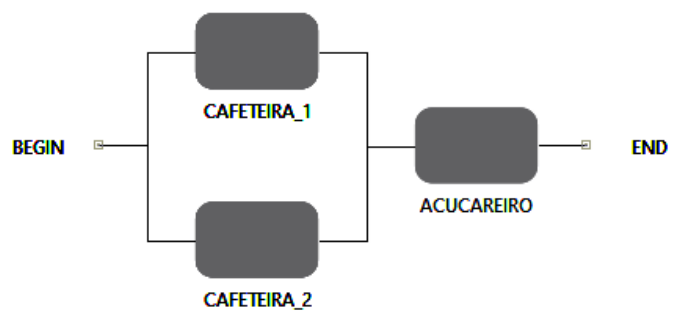

$\left[1-\left(\left(1-D\left(\right.\right.\right.\right.$ cafeteira $\left.\left._{1}\right)\right) *\left(1-D\left(\right.\right.$ cafeteira $\left.\left.\left.\left._{2}\right)\right)\right)\right] * D($ acucareiro $)$

\subsection{Arquitetura Alston}

Alstom é um grupo empresarial multinacional de origem francesa que atua na área de energia e transporte e opera no Brasil há mais de 60 anos (ALSTOM, 2021). Esse grupo é responsável pela linha sul do metrô do Recife que se entende por aproximadamente $14 \mathrm{~km}$, sendo constituído por 12 estações: Recife (RECS), Joana Bezerra (JOAS), Largo da Paz (PAZ), Imbiribeira (IMB), Antônio Falcão (FAL), Shopping (SHO), Tancredo Neves (NEV), Aeroporto (PTO), Porta Larga (LAR), Monte Guararapes (GUA), Prazeres (PRZ) e Cajueiro Seco (CAJ).

As estações RECS, NEV e CAJ são denominadas estações mestre por possuírem a maior parte dos equipamentos de sinalização, como o controlador de movimentação de trens (CMT), o painel de controle local (PCL), o gerenciador de comunicação (GC) e a eletrônica de circuito de via (ECV). As estações que possuem ECV são denominadas estações satélites, e estão presentes nas 3 estações mestre, além de outras 5 estações. Uma estação mestre tem controle sobre determinadas estações. Por exemplo, RECS é a estação mestre das estações RECS, JOAS e PAZ. A Figura 7 apresenta a distribuição de equipamentos nas estações, bem como os respectivos domínios das estações mestre (METROREC, n.d.). 
Figura 7: Disposição dos dispositivos.

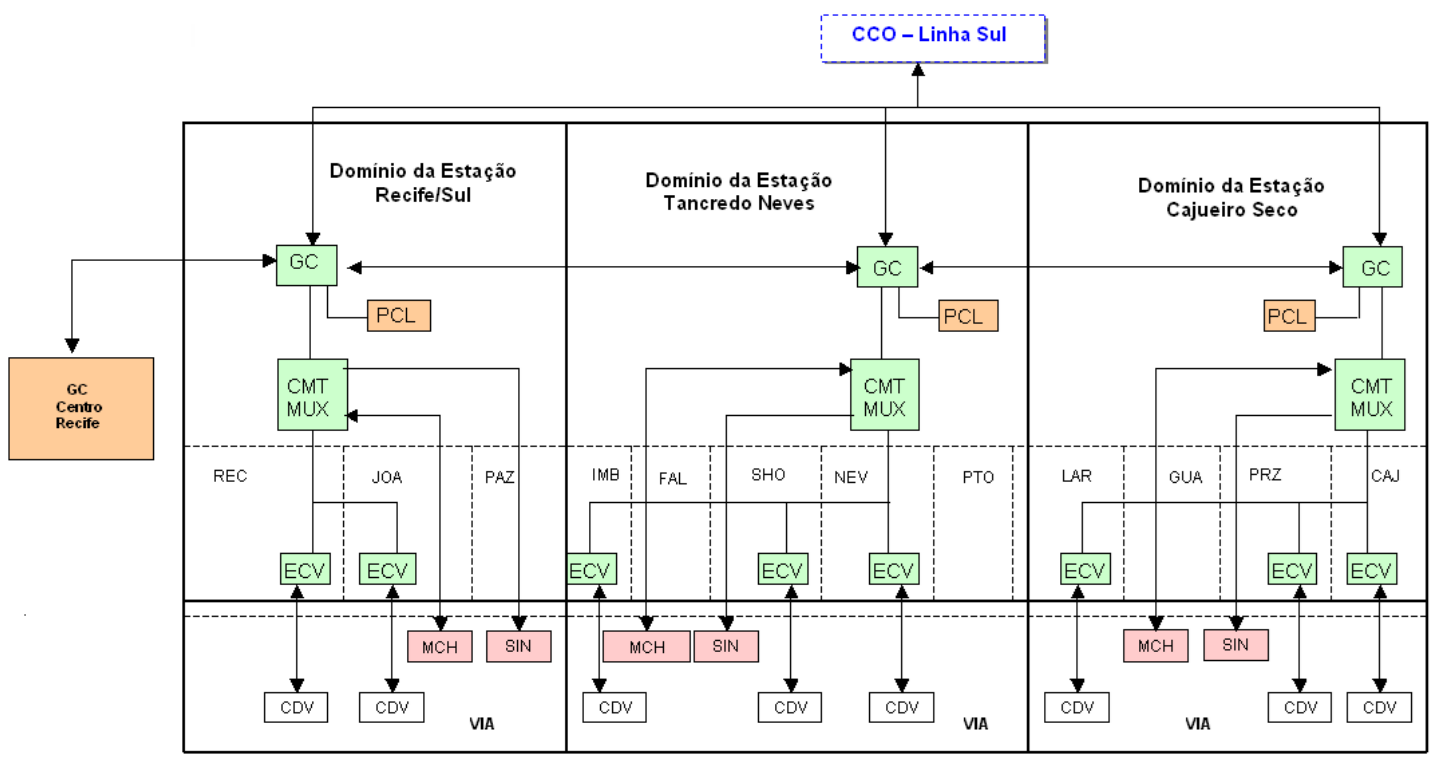

O CMT é o responsável pela conexão entre os equipamentos de campo e também com os ECVs, que são equipamentos que garantem a segurança para a movimentação dos trens. Os ECVs são responsáveis pela detecção dos trens e transmissão dos códigos de velocidade para o trem em um determinado trecho da malha ferroviária, denominado circuito de via (CDV). O GC é responsável pela comunicação entre os equipamentos, recebendo e enviando dados do PCL e transmitindo para os CMTs (METROREC, n.d.).

A localização dos trens é de suma importância para evitar colisões. Na linha sul do metrô do Recife isso é feito através da sinalização de ocupação em um determinado CDV para um ECV. O CDV pode sinalizar os estados OCUPADO, quando houver um trem ocupando o CDV, e DESOCUPADO, quando não houver trens ocupando o CDV, para o ECV. Quando algum desses equipamentos apresenta algum tipo de defeito, é possível que o sistema erroneamente acuse o estado OCUPADO sem que haja trem no CDV, gerando assim uma falsa ocupação (FO).

\section{Trabalhos Relacionados}

Essa seção apresenta artigos relacionados à avaliação de desempenho e de disponibilidade de sistemas ferroviários. A metodologia selecionada para elencar os estudos aqui dispostos focou em apresentar trabalhos que utilizam técnicas de avaliação de desempenho de sistemas (ADS) e de performabilidade para propor estratégias que possam otimizar parâmetros analisados em sistemas ferroviários.

Hai et al. (2019) conduziram um estudo para avaliar a importância da sincronização ferroviária e sua respectiva importância na rede de comunicação do sistema. A pesquisa realizada pelos autores destaca que é de grande importância a execução de um estudo sobre a confiabilidade do cronômetro ao realizar a sincronização de tempo. O trabalho de pesquisa dos autores mostrou que os principais relógios de todos os níveis, com alta confiabilidade, podem atender aos requisitos da fonte de relógio da rede de sincronização da hora ferroviária.

Songwiroj et al. (2018) propuseram uma alternativa para gerar modelos formais de redes ferroviárias a partir dos blocos de construção de redes de Petri coloridas cronometradas. Em vez de desenvolver um 
modelo formal de rede ferroviária a partir do zero usando o CPN Tools, os autores elaboram um conjunto de blocos de construção predefinidos de componentes de rede ferroviária de alto nível, que inclui um bloco de construção da estação de trem, um bloco de construção do trem etc. Além disso, as regras de mapeamento geram automaticamente uma rede ferroviária de alto nível em redes de Petri coloridas cronometradas. O modelo formal resultante é analisado usando as ferramentas do CPN para garantir a correção, segurança e fluidez do modelo formal da rede ferroviária.

Billington (Billington et al. 1996) apresentou uma pesquisa utilizando uma rede ferroviária de via única, com um loop de passagem e vários trens que se deslocam na mesma direção. Nesse trabalho, a malha ferroviária é modelada por uma rede de Petri colorida (CPN - Colored Petri Net), diferenciando-se por realizar uma análise usando a ferramenta de software Design - Occurrence Graph Analyzer (OGA).

Zimmermann e Hommel (2003) avaliou o impacto das falhas dos links de comunicação dos trens sobre o desempenho do sistema de trens na Europa. Os autores ainda analisaram o comportamento da recuperação dos dados perdidos nas falhas avaliadas. Para atingir esse objetivo foi proposta a otimização dos links de comunicação avaliados, além da criação de faixas de interoperabilidade dos sistemas de controle de trens da Europa.

Pindarwati et al. (2015), através da aplicação de técnicas de ADS baseadas em índice de inteligência, analisa o benchmarking do sistema de transporte de cinco grandes cidades da Indonésia. Segundo esses autores, a versatilidade do sistema de transporte urbano pode ser constatada através de metodologias bem estabelecidas que possibilitem procedimentos de comparação através de diferentes indicadores de inteligência de transporte, como deteç̧ão, processamento e controle, comunicação, previsão, recuperação e prevenção.

É possível perceber que não foi o foco de nenhuma das pesquisas anteriores o de quantificar o impacto da disponibilidade no desempenho do sistema de transporte ferroviário. Sendo assim, essa abordagem integrada que estima o impacto da disponibilidade no tempo de viagem, por exemplo, é proposta neste artigo a partir de modelos em SPN e RBD.

\section{Metodologia}

Essa seção apresenta a metodologia adotada que tem por objetivo a análise e otimização de sistemas ferroviários quanto ao desempenho e disponibilidade do serviço. A Figura 8 apresenta os passos adotados pela metodologia proposta. A seguir, cada passo será detalhado.

\subsection{Entendimento do Sistema}

O entendimento do sistema corresponde a fase inicial e é focado na fundamentação teórica para um melhor entendimento do funcionamento dos equipamentos do sistema ferroviário, utilizando manuais técnicos e pesquisas relacionadas. Com um entendimento básico das características dos equipamentos, foi então descoberto que os CDVs, bem como a eletrônica de circuito de via (ECV) poderiam ser objeto de estudo por estarem diretamente relacionados com a disponibilidade da malha ferroviária. Vale destacar que a disponibilidade do serviço impacta diretamente na qualidade e no desempenho do sistema. 
Figura 8: Desenho da pesquisa.

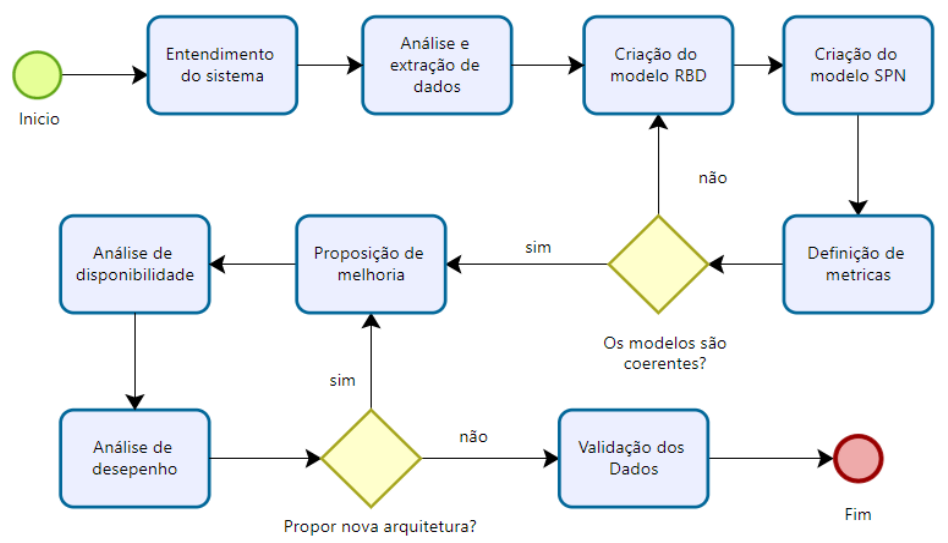

Fonte: Os autores (2021).

\subsection{Análise e Extração de Dados}

Através de uma parceria (ex., com o metrô da cidade do Recife), é possível pegar os dados referentes às solicitações de serviço (SS) relativas aos serviços prestados pelos técnicos para recuperar falhas e colocar o sistema em funcionamento. Os dados fornecidos normalmente são inseridos pelos próprios técnicos e não existe uma padronização sobre o preenchimento das informações. A falta dessa padronização e fiscalização acaba por dificultar a extração de dados. Erros comuns no preenchimento como o de escrever nos lugares errados ou inserção de dados com algum tipo de erro são comuns. Sendo assim, a maior dificuldade pode ser a baixa especificidade na descrição das SS, que muitas vezes se limita em dizer que ocorreu uma SS para um determinado local. Todavia, a partir desses dados é possível extrair os tempos médio de falha ou mean time to failure(MTTF), e o tempo médio de recuperação ou mean time to repair(MTTR) de CDVs presentes no sistema.

\subsection{Criação do Modelos RBD}

A estratégia adotada é a de reduzir o sistema a ser avaliado, reduzindo os trechos das estações analisados. Isso ocorre devido a grande quantidade de componentes que compõem o sistema em estudo e, também, com a finalidade de se ter um maior domínio do sistema em análise. Por isso, apenas uma parte da malha do sistema ferroviário (ex., de Recife) pode vir a ser modelada. Esse trecho analisado se refere ao domínio do controlador de movimentação de trens (CMT) Recife sul (RECS), mas esse sistema é considerado como um sistema completo, com uma via de ida e outra de volta. Esse passo da metodologia corresponde então à criação do modelo RBD para identificar os dispositivos que mais impactam na disponibilidade. Isso foi realizado através do cálculo da importância de cada equipamento na disponibilidade do sistema (Reliability Importance, RI), a finalidade é de identificar os equipamentos críticos, e se propor colocar redundância foi adicionada seguindo os valores de RI em ordem decrescente.

\subsection{Criação do Modelo SPN}

Essa fase tem por objetivo a criação de um modelo de desempenho, em SPN, que represente o comportamento de desempenho e da disponibilidade do sistema ferroviário. Sendo assim, esse modelo 
representará o deslocamento do trem entre as estações e também as mudanças de vias realizadas. Além disso, esse modelo também quantificará o impacto da disponibilidade no desempenho do sistema em análise.

\subsection{Criação das Métricas}

Essa fase tem por objetivo a definição e criação de métricas para avaliar se os modelos estão coerentes com o sistema, bem como quantificar os resultados do processo. Sendo assim, nesta fase são criadas fórmulas para avaliar a disponibilidade e o desempenho do modelo.

\subsection{Proposição de Melhorias}

Essa fase tem por objetivo a modificação teórica do sistema através do modelo para estimar o seu impacto utilizando as métricas previamente definidas. Essa melhoria é feita a partir da proposição de novas arquiteturas com equipamentos redundantes para maximizar a disponibilidade dos equipamentos críticos do sistema.

\subsection{Análise de Disponibilidade}

Essa fase corresponde a execução dos modelos propostos em RBD e em SPN para se computar a disponibilidade. Vale ressaltar que a resposta do modelo RBD pode ser utilizada como parâmetro de entrada para o modelo SPN.

\subsection{Análise de Desempenho}

O modelo de desempenho proposto contemplará não somente a parte de desempenho, mas também a parte de performabilidade. Sendo assim, o modelo SPN de desempenho proposto irá ter algumas partes do modelo de disponibilidade para poder mensurar o impacto da disponibilidade no desempenho.

\subsection{Validação}

Por fim, a última fase corresponde à validação dos resultados obtidos.

\section{Modelos}

Essa seção apresenta os modelos propostos em RBD e em SPN.

\subsection{Modelagem RBD}

A partir de uma planilha de solicitação de serviços (SS) foram coletados diversos dados referentes às falhas dos equipamentos que compõem o sistema ferroviário de Recife. Vale destacar que essa planilha contempla as FOs. Desta planilha foram filtrados os dados para que somente as solicitações de serviços que dizem respeito especificamente a determinado CDV fossem analisadas. Foram, então, extraídos os horários de abertura e encerramento das SSs para, assim, se extrair o MTTR e o MTTF de cada CDV. Devido à complexidade e extensão da malha ferroviária, foi decidido modelar apenas parte dela, especificamente o domínio do CMT de RECS. Desta forma, a malha modelada possui um percurso que sai de RECS, passa por JOAS e chega à PAZ pela VIA 1 e faz o caminho reverso pela VIA 2. Os CDVs pertencentes a VIA 1 possuem o dígito inicial 1, enquanto que os CDVs pertencentes a VIA 2 possuem o dígito inicial 2. Como a planilha de SS possui digitação livre, muitos erros de digitações foram observados, dificultando a identificação de 
campos essenciais, gerando falta de dados para alguns CDVs. Quando um CDV possui dados não disponível, not available (NA), é associado um valor que minimize o impacto deste equipamento no sistema, um MTTR baixo (ex., 1) e um MTTF alto (ex., 100000), considerando que o equipamento falhe raramente e a recuperação seja rápida. A Tabela 2 apresenta os valores de MTTR e MTTF dos CDVs do segmento modelado, e também a estação a que cada CDV pertence. A Figura 9 apresenta o modelo RBD dos CDVs da estação de RECS referentes a VIA 1.

Figura 9: Modelagem em RBD da estação RECS.

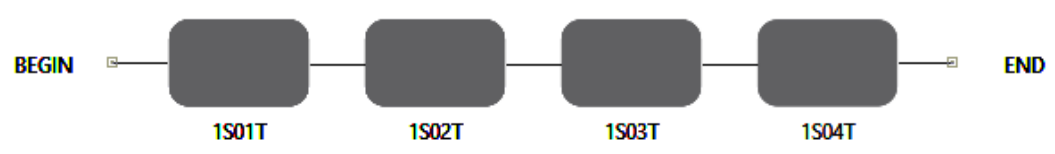

Uma estratégia de redução a fim de simplificar os CDVs presentes entre cada trecho foi adotada de forma que cada estação passa a ser representada por um único bloco. Esse bloco é equivalente ao diagrama de seus CDVs como na Figura 10. Vale destacar que cada modelo RBD com CDVs que representa as estações possui os CDVs conectados em série, pois a falha de um desses equipamentos ocasionará a falha de todo o sistema de detecção de trens. Os valores MTTR e MTTF das estações foram computados a partir de modelos RBD, similares ao da Figura 9 e com os CDVs correspondentes, e estão dispostos na Tabela 3.

Tabela 2: Valores MTTF e MTTR dos CDVs.

\begin{tabular}{|c|c|c|c|c|c|c|}
\hline \multirow{2}{*}{ Estação } & \multicolumn{3}{|c|}{ VIA 1} & \multicolumn{3}{|c|}{ VIA 2} \\
\hline & $C D V$ & MTTR & $M T T F$ & $C D V$ & MTTR & $M T T F$ \\
\hline RECS & 1S01T & $N A$ & $N A$ & $2 \mathrm{~S} 01 \mathrm{~T}$ & $N A$ & $N A$ \\
\hline RECS & $1 \mathrm{~S} 02 \mathrm{~T}$ & 13683 & 944972 & $2 \mathrm{~S} 02 \mathrm{~T}$ & 21993 & 2056279 \\
\hline RECS & 1S03T & 255 & 1097220 & $2 \mathrm{~S} 03 \mathrm{~T}$ & 109004 & 959622 \\
\hline RECS & 1S04T & 132088 & 946424 & $2 \mathrm{~S} 04 \mathrm{~T}$ & 1003 & 1641082 \\
\hline JOAS & $1 \mathrm{~S} 05 \mathrm{~T}$ & 1052 & 1262830 & $2 \mathrm{~S} 05 \mathrm{~T}$ & 1052 & 1262830 \\
\hline JOAS & $1 \mathrm{~S} 06 \mathrm{~T}$ & 65961 & 911872 & $2 \mathrm{~S} 06 \mathrm{~T}$ & 1052 & 1262830 \\
\hline JOAS & 1S07T & 1052 & 1262830 & $2 \mathrm{~S} 07 \mathrm{~T}$ & 1052 & 1262830 \\
\hline JOAS & 1S08T & 1052 & 1262830 & $2 \mathrm{~S} 08 \mathrm{~T}$ & 22091 & 1050718 \\
\hline
\end{tabular}

Tabela 3: Valores MTTF e MTTR das estações após o cálculo.

\begin{tabular}{|c|c|c|c|c|}
\hline \multirow{2}{*}{ Estação } & \multicolumn{2}{|c|}{ VIA 1} & \multicolumn{2}{c|}{ VIA 2 } \\
\cline { 2 - 5 } & $M T T R$ & $M T T F$ & $M T T R$ & $M T T F$ \\
\hline RECS & 51659 & 330443 & 66743 & 376158 \\
\hline JOAS & 21604 & 287996 & 7086 & 300539 \\
\hline
\end{tabular}

A fim de aumentar a disponibilidade da malha ferroviária, foi proposto a criação de rotas alternativas (RAs) para os CDVs que são mais representativos para a disponibilidade do sistema analisado. Para isso, as RAs são implementadas seguindo a ordem do índice da importância para a disponibilidade (AI). As RAs podem ser representadas como uma duplicação de um ou mais CDVs. Neste trabalho, assumiu-se que as RAs possuem os mesmos parâmetros do CDV original. A Figura 11 mostra a representação em RBD de uma RA do CDV 1S03T da estação RECS. 
Figura 11: Representação de RAs em RBD.

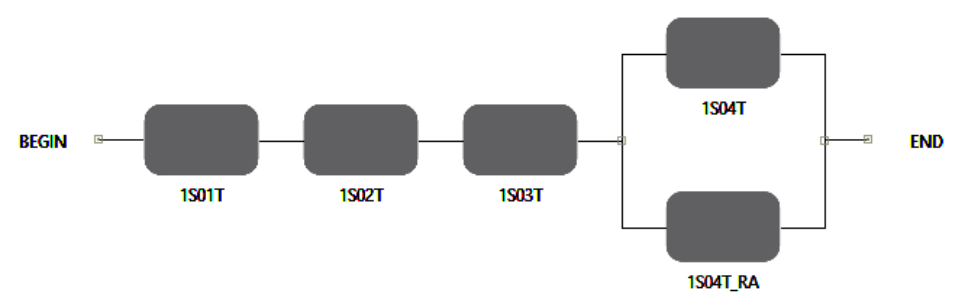

\subsection{Modelagem $S P N$}

Para representar o funcionamento e a movimentação do trem na malha, foi criado um modelo em SPN. A Figura 12 apresenta uma parte da malha modelada em SPN, referente ao trecho entre as estações RECS_1 e JOAS_1. Nesse modelo, é possível verificar os CDVs referentes à estação RECS. No modelo SPN disposto na Figura 12, o token começa inicialmente em RECS_1, é possível disparar a transição T0 que vai consumir o token presente em RECS_1, e gera um token em CDV_1S01T. O disparo da transição T0 representa então uma pequena movimentação do trem saindo de RECS_1 e chegando em CDV_1S01T, parte intermediária do trecho entre RECS_1 e JOAS_1.

Figura 12: Representação de parte da malha em SPN.

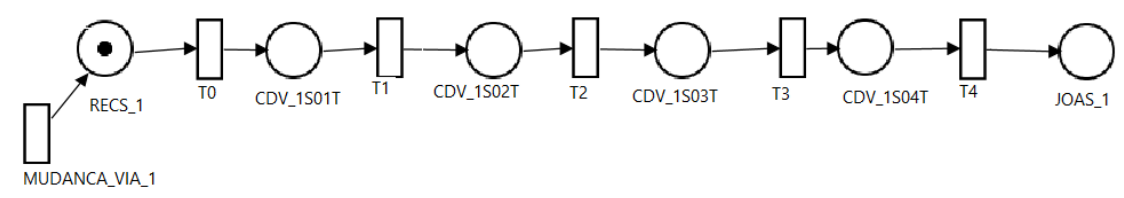

As RAs também podem ser representadas em SPN com adição de um lugar e duas transições representando as RAs. Quando o trem se encontra no CDV imediatamente anterior, ele pode continuar na rota principal ou pegar um desvio pela RA. Vale destacar que estamos considerando uma situação em que ambos os trajetos são equivalentes em distância e em velocidade média. Sendo assim, a adição de uma rota alternativa acaba por aumentar a disponibilidade do sistema. A Figura 13 apresenta a modelagem de uma parte da malha com a presença de uma RA, representada pelo caminho da transição T6, do lugar CDV_1S04T_RA e da transição T7.

Figura 13: Representação de uma RA em SPN.

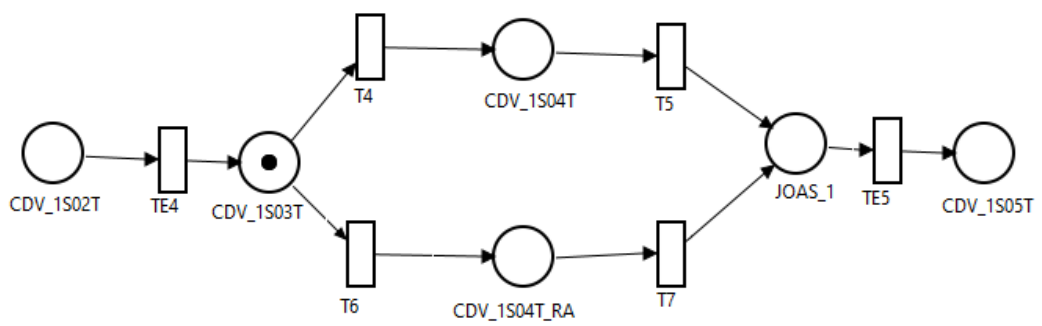

As RAs são equivalentes em distância. Sendo assim, a adição de uma RA afeta apenas a disponibilidade. O modelo SPN proposto utiliza o tempo médio que cada trem leva para ir de uma estação a outra. Desta forma, no modelo SPN, as estações são representadas como lugares e com transições que 
representam o movimento dos trens entre as estações. A Figura 14 mostra o modelo SPN proposto para representar o deslocamento do trem entre as estações. É possível simular a viagem completa do trem passando por JOAS_1 (disparo da transição T1) e chegando a PAZ_1 (disparo da transição T2). Em seguida, de PAZ_1 para PAZ_2 (disparo de T3); de PAZ_2 passando por JOAS_2 (disparo de T4); JOAS_2 chegando em RECS_2 (disparo de T5); e de RECS_2 ao estado inicial (disparo de T0).

Figura 14: Representação da malha reduzida em SPN.

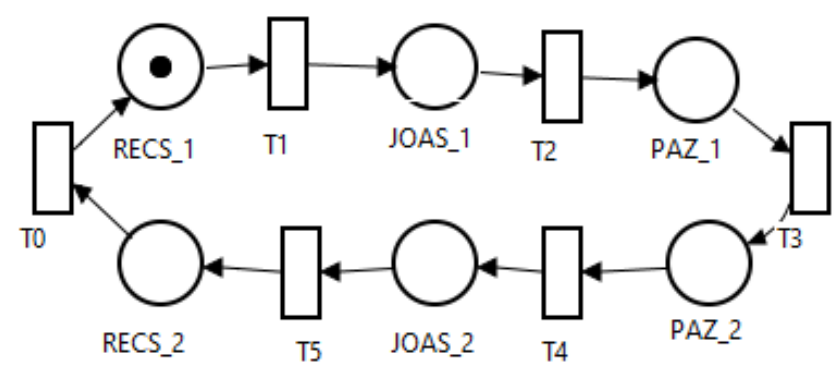

O modelo representado na Figura 14 simula a movimentação do trem de forma simplificada, assumindo que nunca haverá defeitos impedindo a movimentação do trem e sem tratamento para evitar colisões entre trens. Para representar o impacto da disponibilidade de um trecho no desempenho do sistema, foram adicionadas sub-redes que representam o comportamento do sistema quanto a disponibilidade. Para evitar colisões de trens, foram utilizados arcos inibidores de forma que a transição que representa a movimentação do trem entre estações só pode ser disparada quando não existe nenhum trem na estação destino. A Figura 15 representa esse modelo. Nessa figura, as transições XX_FAIL e XX_REPAIR, onde XX assume o nome da estação (ex., RECS1), têm respectivamente os tempos MTTF e MTTR presentes na Tabela 3. O tempo médio de viagem entre as estações (representado em minutos) está presente na Tabela 4. Essa tabela também apresenta a distância (em metros) existente entre cada uma das estações analisadas. O tempo das transições de troca de via adotado foi de 5 minutos, e está associado às transições T6 e T3. O tempo médio de viagem (um ciclo completo) pode ser calculado conforme mostrado na Equação 9.

Tabela 4: Tempo médio de viagem entre as estações.

\begin{tabular}{|c|c|c|c|}
\hline \multirow{2}{*}{ Estações } & \multirow{2}{*}{ Extensão } & \multicolumn{2}{|c|}{ Tempo de viagem } \\
\cline { 3 - 4 } & & VIA 1 & VIA 2 \\
\hline RECS - JOAS & 1284,974 & 1,606 & 2,409 \\
\hline JOAS - PAZ & 1486,586 & 1,858 & 1,820 \\
\hline
\end{tabular}

$\underline{E}\left\{R E C S_{1}\right\}+E\left\{R E C S_{2}\right\}+E\left\{O A S_{1}\right\}+E\left\{\mid O A S_{2}\right\}+E\left\{P A Z_{1}\right\}+E\left\{P A Z_{2}\right\}$

$$
\left(\frac{E\left\{P A Z_{1}\right\}}{5}\right)
$$


Figura 15: Modelo SPN da malha ferroviária.

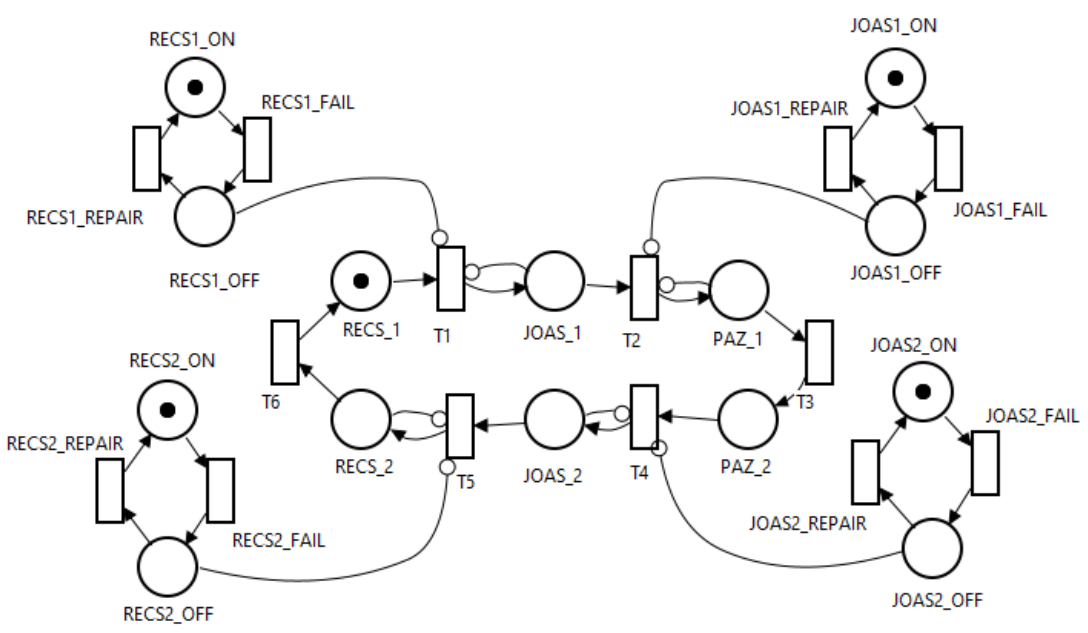

\section{Estudo de Caso}

Essa seção apresenta um estudo de caso para ilustrar a aplicabilidade do modelo SPN proposto para calcular o aumento da disponibilidade pela adição de componentes redundantes. Utilizando o modelo descrito na Figura 15, é possível utilizar os MTTR e MTTFs para estimar o impacto da disponibilidade no desempenho do sistema do metrô.

\subsection{Utilizando AI dos CDVS para maximizar a disponibilidade}

Com a finalidade de maximizar a disponibilidade do sistema, foi criado um modelo RBD, contendo todos os CDVs do segmento modelado, para fazer o cálculo da AI e, então, adicionar RAs ao CDVs de acordo com o seu impacto na disponibilidade. A Tabela 5 apresenta os valores de AI para cada interação, onde a arquitetura 0 corresponde ao baseline e não apresenta rotas alternativas. A partir dessa arquitetura, RAs são adicionadas de acordo com o AI. Por exemplo, a arquitetura 1 corresponde a arquitetura 0 com o CDV1S04T replicado. Isso ocorreu pelo fato deste dispositivo ser o de valor mais elevado conforme mostrado na Tabela 5. A Figura 16 apresenta a disponibilidade calculada para cada uma das arquiteturas.

Tabela 5: Medição de AI dos CDVs.

\begin{tabular}{|c|c|c|c|c|l|}
\hline \multirow{2}{*}{ Arquitetura } & \multicolumn{2}{|l|}{ Importância do $C D V$ na disponibilidade. } & \multirow{2}{*}{$C D V$ s com $R A$ s } \\
\cline { 2 - 5 } & $1 \mathrm{~S} 04 \mathrm{~T}$ & $2 \mathrm{~S} 03 \mathrm{~T}$ & $1 \mathrm{~S} 06 \mathrm{~T}$ & $2 \mathrm{~S} 08 \mathrm{~T}$ & \\
\hline 0 & 0,7954 & 0,7772 & 0,7484 & 0,7126 & \\
\hline 1 & 0,0974 & 0,8724 & 0,8401 & 0,7999 & $1 \mathrm{~S} 04 \mathrm{~T}$ \\
\hline 2 & 0,1073 & 0,0889 & 0,9258 & 0,8815 & $1 \mathrm{~S} 04 \mathrm{~T}, 2 \mathrm{~S} 03 \mathrm{~T}$ \\
\hline 3 & 0,1145 & 0,0949 & 0,0624 & 0,9410 & $1 \mathrm{~S} 04 \mathrm{~T}, 2 \mathrm{~S} 03 \mathrm{~T}, 1 \mathrm{~S} 06 \mathrm{~T}$ \\
\hline 4 & 0,1169 & 0,0969 & 0,0637 & 0,0193 & $1 \mathrm{~S} 04 \mathrm{~T}, 2 \mathrm{~S} 03 \mathrm{~T}, 1 \mathrm{~S} 06 \mathrm{~T}, 2 \mathrm{~S} 08 \mathrm{~T}$ \\
\hline
\end{tabular}

Figura 16: Disponibilidade $\mathrm{x}$ arquitetura.

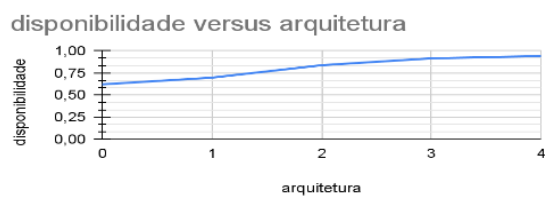

Quando apenas um trem está circulando nas vias, não existe o problema de congestionamento. No entanto, falhas dos trechos imediatamente à frente podem impedir o trem de seguir viagem. Quando existe 
mais de um trem, o problema do congestionamento surge. Falhas em outros pontos do trajeto também podem afetar a movimentação do trem, pois se um trem ficar parado em uma estação, um trem imediatamente atrás não poderá avançar. A Figura 17 mostra os resultados obtidos, assumindo dois trens na malha.

Figura 17: Tempo médio de viagem $\mathrm{x}$ disponibilidade.

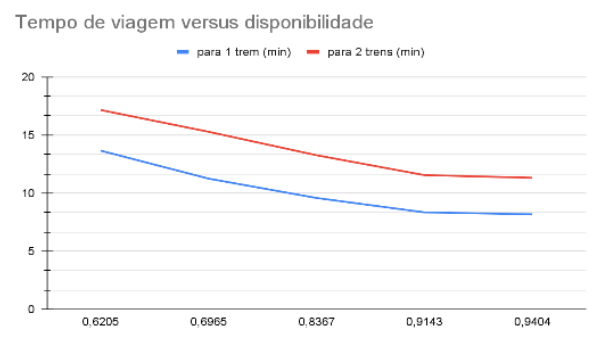

\subsection{Utilizando AI das estações para maximizar a disponibilidade}

Em uma abordagem diferente, o modelo RBD pode ser utilizado para adicionar RAs a todo percurso entre as estações, em vez de apenas CDVs críticos e se quantificar o impacto direto na disponibilidade e desempenho do sistema. A Tabela 6 apresenta os valores de AI das estações para cada interação.

Figura 18 mostra uma comparação entre as duas abordagens no ganho de disponibilidade. O ganho na disponibilidade ao adicionar RAs em todo percurso em relação aos CDVs mais impactantes não é significativo. Por se tratar de um trajeto maior, o que implicaria num custo maior.

Tabela 6: Medição de AI das estações.

\begin{tabular}{|c|c|c|c|c|l|}
\hline \multirow{2}{*}{ Arquitetura } & \multicolumn{4}{|c|}{ Importância da estação na disponibilidade. } & \multirow{2}{*}{ Estações com $R A$ 's } \\
\cline { 2 - 5 } & $R E C S_{1}$ & $R E C S_{2}$ & $J O A S_{1}$ & $J O A S_{2}$ & \\
\hline 0 & 0,7718 & 0,7859 & 0,7175 & 0,6832 & \\
\hline 1 & 0,8881 & 0,1184 & 0,8256 & 0,7861 & RECS \\
\hline 2 & 0,1200 & 0,1344 & 0,9373 & 0,8924 & RECS $_{2}$, RECS $_{1}$ \\
\hline 3 & 0,1284 & 0,1438 & 0,0654 & 0,9547 & RECS $_{2}$, RECS $_{1}$, JOAS $_{1}$ \\
\hline 4 & 0,1314 & 0,1471 & 0,0669 & 0,0219 & RECS $_{2}$, RECS $_{1}$, JOAS $_{1}$, JOAS $_{2}$ \\
\hline
\end{tabular}

Figura 18: Comparação no ganho de disponibilidade

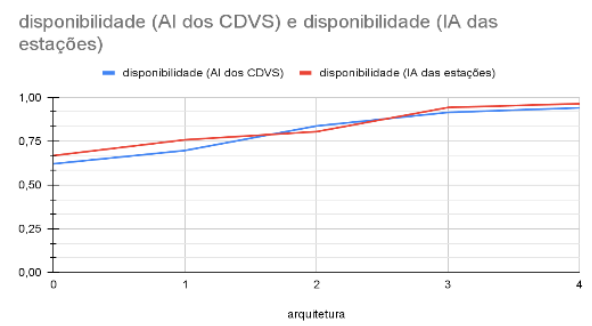

Para o trecho modelado, com a adição de quatro RAs foi possível obter um aumento de $44 \%$ na disponibilidade dos CDVs. Este aumento na disponibilidade gerou uma redução no tempo médio de viagem de $51 \%$. Os modelos criados possibilitaram a identificação dos CDVs mais impactantes no desempenho e calcular o impacto das mudanças propostas. A diferença no ganho de desempenho entre as duas abordagens foi inferior a $3 \%$.

\section{Considerações finais}

Esse trabalho propôs um conjunto de modelos para a avaliação integrada de desempenho e disponibilidade de sistemas ferroviários. $\mathrm{O}$ artigo demonstrou a aplicabilidade dos modelos propostos em um estudo de caso levando em consideração o sistema ferroviário da cidade de Recife. Os modelos se mostraram 
eficientes para representar o sistema analisado e propôs melhorias significativas no sistema, onde foi possível melhorar em aproximadamente $50 \%$ a disponibilidade do serviço de metrô da cidade analisada. Além disso, também foi possível quantificar o impacto dessa melhoria na disponibilidade no tempo de viagem, que foi melhorada em 40\%. Como futuro direcionamento desta pesquisa, pretende-se modelar a malha ferroviária da cidade de Recife por completo. Além disso, uma melhor identificação de falhas será proposta nos trabalhos futuros levando em consideração outros tipos de falhas e utilizando abordagens diferentes.

\section{Agradecimentos}

Os autores gostariam de agradecer ao CNPq e a FACEPE pelo apoio financeiro para a realização desta pesquisa.

\section{Referências}

ALSTOM (2020). Alstom celebra 65 anos de presença no brasil com a chegada de projetos para produção nacional de trens para o país e o mundo.”, Janeiro, Online, acessado em 1 de abril de 2021. Disponível em: https: //www.alstom.com/pt/alstom-no-brasil.

BILLINGTON, J.; JANCZURA, C. (1996). State space analysis of a railway network. IEEE International Conference on Systems, Man and Cybernetics. Information Intelligence and Systems (Cat. No.96CH35929), volume 3, pages 2386-2391.

CBTU (2018). Metrorec, unidade operacional que atende diretamente os municípios do recife, cabo, jaboatão dos guararapes e camaragibe e, indiretamente, os demais municípios da região metropolitana, Abril 2018. Online, acessado em 1 de abril de 2021. Disponível em: https://www.cbtu.gov.br/index.php/pt/sistemas-cbtu/

HAI, N.; ZHANG, C.; YU, Y.; TANG, Y. (2019). Reliability analysis of clock source of railway time synchronization network based on SPN. In 2019 International Conference on Intelligent Transportation, Big Data Smart City (ICITBS), pages 45-48.

MACIEL, P. R.; TRIVEDI, K. S.; MATIAS, R.; KIM, D. S. (2012). Dependability modeling, Performance and Dependability in Service Computing: Concepts, Techniques and Research Directions. IGI Global, 2012, ch. 3.

MACIEL, P. R.; LINS, R. D.; CUNHA, P. R. (1996). Introdução a redes de Petri e aplicações. Instituto de Computação - UNICAMP, ch. 4.4, pp. 125-130.

METROREC (n.d.). MAM - manual de manutenção do equipamento CMT. alstom, ch. 2, pp. 8-13.

PINDARWATI, A.; WIJAYANTO, A.W. (2015). Measuring performance level of smart transportation system in big cities of Indonesia comparative study: Jakarta, Bandung, Medan, Surabaya, and Makassar, International Conference on Information Technology Systems and Innovation (ICITSI), 2015, pp. 1-6, doi: 10.1109/ICITSI.2015.7437716.

SILVA, B.; MATOS, R.; CALLOU, G.; FIGUEIREDO, J.; DANTAS, J.; LOBO JR, A.; ALVES, V.; MACIEL, P. (2015). Mercury: An Integrated Environment for Performance and Dependability Evaluation of 
General Systems. In: Industry Track at 45th Dependable Systems and Networks Conference, 2015, Rio de Janeiro. Proceedings of 45th Dependable Systems and Networks Conference, 2015. v. v. p. 1-6.

SONGWIROJ, N.; VATANAWOOD, W.; VANIT-ANUNCHAI, S. (2018). Railway network modeling using building block of timed coloured petri nets. In 2018 IEEE 4th International Conference on Computer and Communications (ICCC), pages 528-533.

ZIMMERMANN, A.; HOMMEL, G. (2003). A train control system case study in model-based real time system design. Proceedings International Parallel and Distributed Processing Symposium, page 8 pp. 